\title{
Cocrystal and salt forms of furosemide: solubility and diffusion variations
}

\section{Manas Banik, ShanmukhaPrasad Gopi, Somnath Ganguly and Gautam R. Desiraju*}

Solid State and Structural Chemistry Unit, Indian Institute of Science, Bangalore 560 012, India. Fax: +91 80 23602306; Tel: +91 80 22933311; E-mail: desiraju@sscu.iisc.ernet.in

\section{Supporting Information}

\section{Contents}

\begin{tabular}{|l|l|}
\hline Description & Page No. \\
\hline Table S1. Neutron normalized hydrogen bond geometrical parameters & S2-S3 \\
\hline Table S2. Melting points of salt/cocrystal/coformer/API & S4 \\
\hline Table S3. pH variation after solubility experiments. & S4 \\
\hline $\begin{array}{l}\text { Figure S1. ORTEP diagrams of FSM-TOL-MeOH, FSM-TOL-MeNO } 2 \text { and FSM- } \\
\text { toluene }\end{array}$ & S5 \\
\hline Figure S2. Powder x-ray diffraction patterns of new solid forms of FSM & S6-S10 \\
\hline Figure S3. DSC and TGA of FSM and its salt/cocrystals & S11-S14 \\
\hline Figure S4. FT-IR spectra of FSM and its salt/cocrystals & S14-S17 \\
\hline Figure S5. Overlay diagrams of FSM conformations. & $\mathrm{S} 18$ \\
\hline
\end{tabular}


Table S1. Neutron normalized hydrogen bond geometrical parameters (intermolecular O-H, N-H and $\mathrm{C}-\mathrm{H}$ distances).

\begin{tabular}{|c|c|c|c|c|c|c|}
\hline Compound & Interaction & $\begin{array}{l}\mathrm{D}-\mathrm{H} / \\
\AA\end{array}$ & $\begin{array}{l}\mathrm{H} \cdots \mathrm{A} / \\
\AA\end{array}$ & $\mathrm{D} \cdots \mathrm{A} / \AA$ & $\underset{\mathrm{o}}{\angle \mathrm{D}-\cdots \mathrm{A} /}$ & Symmetry \\
\hline \multirow{5}{*}{$\begin{array}{l}\text { FSM- } \\
\text { TMPZ }\end{array}$} & $\mathrm{O}(1)-\mathrm{H}(1 \mathrm{O}) \cdots \mathrm{N}(3)$ & 0.98 & 1.73 & $2.670(5)$ & 159 & \\
\hline & $\mathrm{N}(2)-\mathrm{H}(2 \mathrm{~A}) \cdots \mathrm{O}(5)$ & 1.01 & 2.43 & $3.152(6)$ & 128 & $\mathrm{x}, 1+\mathrm{y}, \mathrm{z}$ \\
\hline & $\mathrm{N}(2)-\mathrm{H}(2 \mathrm{~B}) \cdots \mathrm{O}(5)$ & 1.01 & 2.05 & $2.973(7)$ & 152 & $1-x, 1 / 2+y, 1 / 2-z$ \\
\hline & $\mathrm{C}(15)-(15 \mathrm{~A}) \cdots \mathrm{O}(1)$ & 1.08 & 2.28 & $3.198(7)$ & 141 & \\
\hline & $\mathrm{C}(16) \mathrm{H}(16 \mathrm{~B}) \cdots \mathrm{O}(2)$ & 1.08 & 2.28 & $3.353(7)$ & 170 & \\
\hline \multirow[t]{7}{*}{ FSM-ANT } & $\mathrm{N}(1)-\mathrm{H}(1 \mathrm{~A}) \cdots \mathrm{O}(3)$ & 1.01 & 2.28 & $2.965(4)$ & 124 & $-1 / 2+x, 1 / 2+y, z$ \\
\hline & $\mathrm{O}(1)-\mathrm{H}(1 \mathrm{O}) \cdots \mathrm{O}(6)$ & 0.98 & 1.59 & $2.557(4)$ & 166 & $1 / 2+x, 1 / 2+y, z$ \\
\hline & $\mathrm{N}(2)-\mathrm{H}(2 \mathrm{~A}) \cdots \mathrm{N}(3)$ & 1.01 & 2.08 & $3.086(5)$ & 172 & \\
\hline & $\mathrm{N}(2)-\mathrm{H}(2 \mathrm{~B}) \cdots \mathrm{O}(5)$ & 1.01 & 2.25 & $3.123(4)$ & 145 & $1 / 2+x,-1 / 2+y, z$ \\
\hline & $\mathrm{N}(3)-\mathrm{H}(3 \mathrm{~A}) \cdots \mathrm{O}(4)$ & 1.01 & 2.02 & $3.004(4)$ & 164 & $2-x, y, 1 / 2-z$ \\
\hline & $\mathrm{N}(4)-\mathrm{H}(4 \mathrm{~A}) \cdots \mathrm{O}(2)$ & 1.01 & 1.98 & $2.967(5)$ & 165 & $-1 / 2+x,-1 / 2+y, z$ \\
\hline & $\mathrm{C}(8)-\mathrm{H}(8 \mathrm{~A}) \cdots \mathrm{O}(3)$ & 1.08 & 2.44 & $3.149(4)$ & 122 & $-1 / 2+x, 1 / 2+y, z$ \\
\hline \multirow{7}{*}{$\begin{array}{l}\text { FSM-PPZ } \\
(1: 1)\end{array}$} & $\mathrm{N}(2)-\mathrm{H}(2 \mathrm{~A}) \cdots \mathrm{O}(1)$ & 1.01 & 1.82 & $2.822(3)$ & 171 & $1-x, 1-y,-z$ \\
\hline & $\mathrm{N}(2)-\mathrm{H}(2 \mathrm{~B}) \cdots \mathrm{O}(1)$ & 1.01 & 2.11 & $2.909(3)$ & 134 & $\mathrm{x},-1+\mathrm{y}, \mathrm{z}$ \\
\hline & $\mathrm{N}(4)-\mathrm{H}(4 \mathrm{~A}) \cdots \mathrm{O}(2)$ & 1.01 & 1.68 & $2.666(3)$ & 166 & $1-x, 2-y,-z$ \\
\hline & $\mathrm{N}(4)-\mathrm{H}(4 \mathrm{~B}) \cdots \mathrm{N}(3)$ & 1.01 & 1.82 & $2.806(3)$ & 163 & $\begin{array}{l}1 / 2-x, 1 / 2+y,-1 / 2- \\
z\end{array}$ \\
\hline & $\mathrm{C}(8)-\mathrm{H}(8 \mathrm{~B}) \cdots \mathrm{O}(5)$ & 1.08 & 2.36 & $3.337(4)$ & 149 & $\begin{array}{l}1 / 2-x, 1 / 2+y, 1 / 2- \\
z\end{array}$ \\
\hline & $\mathrm{C}(12)-\mathrm{H}(12) \cdots \mathrm{O}(1)$ & 1.08 & 2.31 & $3.395(4)$ & 178 & $1 / 2+x, 3 / 2 y, 1 / 2+z$ \\
\hline & $\mathrm{C}(16)-(16 \mathrm{~A}) \cdots \mathrm{O}(4)$ & 1.08 & 2.36 & $3.176(3)$ & 131 & \\
\hline \multirow{6}{*}{$\begin{array}{l}\text { FSM-PCM } \\
\text { hydrate }\end{array}$} & $\mathrm{N}(1)-\mathrm{H}(1 \mathrm{~A}) \cdots \mathrm{O}(3)$ & 1.01 & 2.09 & $3.031(8)$ & 154 & \\
\hline & $\mathrm{N}(1) \mathrm{H}(1 \mathrm{~B}) \cdots \mathrm{N}(2)$ & 1.01 & 2.21 & $3.108(10)$ & 148 & $-3-x, 1-y, 1-z$ \\
\hline & $\mathrm{O}(2)-\mathrm{H}(1 \mathrm{O}) \cdots \mathrm{O}(1)$ & 0.98 & 1.57 & $2.551(7)$ & 173 & \\
\hline & $\mathrm{C}(3)-\mathrm{H}(3) \cdots \mathrm{O}(100)$ & 1.08 & 2.42 & $3.24(3)$ & 131 & $-2-x,-y, 1-z$ \\
\hline & $\mathrm{C}(4)-\mathrm{H}(4) \cdots \mathrm{O}(6)$ & 1.08 & 2.42 & $3.306(14)$ & 138 & $-3-x,-y, 1-z$ \\
\hline & $\mathrm{C}(4)-\mathrm{H}(4) \cdots \mathrm{O}\left(6^{\prime}\right)$ & 1.08 & 2.19 & $3.270(13)$ & 177 & $-3-x,-y, 1-z$ \\
\hline \multirow{8}{*}{$\begin{array}{l}\text { FSM-TOL- } \\
\text { EtOH }\end{array}$} & $\mathrm{O}(1)-\mathrm{H}(1 \mathrm{O}) \cdots \mathrm{O}(6)$ & 0.98 & 1.59 & $2.563(3)$ & 172 & \\
\hline & $\mathrm{N}(2)-\mathrm{H}(2 \mathrm{~A}) \cdots \mathrm{O}(5)$ & 1.01 & 1.96 & $2.956(3)$ & 167 & $-x,-1 / 2+y, 3 / 2-z$ \\
\hline & $\mathrm{N}(2)-\mathrm{H}(2 \mathrm{~B}) \cdots \mathrm{O}(7)$ & 1.01 & 1.84 & $2.800(4)$ & 159 & \\
\hline & $\mathrm{N}(3)-\mathrm{H}(3 \mathrm{~A}) \cdots \mathrm{O}(2)$ & 1.01 & 1.93 & $2.899(3)$ & 161 & \\
\hline & $\mathrm{N}(3)-\mathrm{H}(3 \mathrm{~B}) \cdots \mathrm{N}(2)$ & 1.01 & 2.37 & $3.284(3)$ & 151 & $\mathrm{x}, 1 / 2-\mathrm{y},-1 / 2+\mathrm{z}$ \\
\hline & $\mathrm{O}(7)-\mathrm{H}(7 \mathrm{O}) \cdots \mathrm{O}(4)$ & 0.98 & 1.87 & $2.825(3)$ & 163 & $-x, 1 / 2+y, 3 / 2-z$ \\
\hline & $\mathrm{C}(10)-\mathrm{H}(10) \cdots \mathrm{O}(4)$ & 1.08 & 2.46 & $3.423(3)$ & 148 & $-\mathrm{x}, 1-\mathrm{y}, 1-\mathrm{z}$ \\
\hline & $\mathrm{C}(12)-\mathrm{H}(12) \cdots \mathrm{O}(6)$ & 1.08 & 2.31 & $3.229(3)$ & 142 & $1+x, y, z$ \\
\hline
\end{tabular}




\begin{tabular}{|c|c|c|c|c|c|c|}
\hline & $\mathrm{C}(15)-\mathrm{H}(15) \cdots \mathrm{O}(5)$ & 1.08 & 2.51 & $3.433(3)$ & 143 & $\mathrm{x}, 1 / 2-\mathrm{y},-1 / 2+\mathrm{z}$ \\
\hline \multirow{9}{*}{$\begin{array}{l}\text { FSM-TOL- } \\
\mathrm{MeOH}\end{array}$} & $\mathrm{O}(1)-\mathrm{H}(1 \mathrm{O}) \cdots \mathrm{O}(6)$ & 0.98 & 1.57 & $2.542(2)$ & 171 & \\
\hline & $\mathrm{N}(2)-(2 \mathrm{~A}) \cdots \mathrm{O}(10)$ & 1.01 & 1.83 & $2.746(13)$ & 149 & $2-x,-1 / 2+y, 1 / 2-z$ \\
\hline & $\mathrm{N}(2)-\mathrm{H}(2 \mathrm{~B}) \cdots \mathrm{O}(4)$ & 1.01 & 157 & $2.941(2)$ & 157 & $2-x, 1 / 2+y, 1 / 2-z$ \\
\hline & $\mathrm{N}(3)-\mathrm{H}(3 \mathrm{~A}) \cdots \mathrm{O}(2)$ & 1.01 & 1.93 & $2.893(3)$ & 159 & \\
\hline & $\mathrm{N}(3)-\mathrm{H}(3 \mathrm{~B}) \cdots \mathrm{N}(2)$ & 1.01 & 2.42 & $3.352(3)$ & 154 & $\mathrm{x}, 1 / 2-\mathrm{y},-1 / 2+\mathrm{z}$ \\
\hline & $\mathrm{O}(10)-(10 \mathrm{~A}) \cdots \mathrm{O}(5)$ & 0.98 & 1.95 & $2.852(5)$ & 152 & \\
\hline & $\mathrm{C}(10)-\mathrm{H}(10) \cdots \mathrm{O}(5)$ & 1.08 & 2.47 & $3.490(3)$ & 156 & $2-x,-y,-z$ \\
\hline & $\mathrm{C}(12)-\mathrm{H}(12) \cdots \mathrm{O}(6)$ & 1.08 & 2.35 & $3.175(3)$ & 132 & $1+\mathrm{x}, \mathrm{y}, \mathrm{z}$ \\
\hline & $\mathrm{C}(21)-(21 \mathrm{~B}) \cdots \mathrm{O}(1)$ & 1.08 & 2.50 & $3.522(4)$ & 157 & \\
\hline \multirow{5}{*}{$\begin{array}{l}\text { FSM-TOL- } \\
\mathrm{MeNO}_{2}\end{array}$} & $\mathrm{O}(1)-\mathrm{H}(1 \mathrm{O}) \cdots \mathrm{O}(6)$ & 0.98 & 1.61 & $2.579(5)$ & 166 & $1-x,-y,-z$ \\
\hline & $\mathrm{N}(2)-\mathrm{H}(2 \mathrm{~A}) \cdots \mathrm{O}(4)$ & 1.01 & 1.91 & $2.918(7)$ & 174 & $2-x,-1 / 2+y, 1 / 2-z$ \\
\hline & $\mathrm{N}(2)-\mathrm{H}(2 \mathrm{~B}) \cdots \mathrm{O}(8)$ & 1.01 & 2.20 & $3.070(7)$ & 143 & $1-\mathrm{x},-1 / 2+\mathrm{y}, 1 / 2-\mathrm{z}$ \\
\hline & $\mathrm{N}(3)-\mathrm{H}(3 \mathrm{~A}) \cdots \mathrm{N}(2)$ & 1.01 & 2.37 & $3.338(6)$ & 160 & $1-\mathrm{x}, 1 / 2+\mathrm{y}, 1 / 2-\mathrm{z}$ \\
\hline & $\mathrm{N}(3)-\mathrm{H}(3 \mathrm{~B}) \cdots \mathrm{O}(2)$ & 1.01 & 1.93 & $2.929(5)$ & 170 & $1-\mathrm{x},-\mathrm{y},-\mathrm{z}$ \\
\hline \multirow{3}{*}{$\begin{array}{l}\text { FSM- } \\
\text { toluene }\end{array}$} & $\mathrm{N}(2)-\mathrm{H}(1 \mathrm{~B}) \cdots \mathrm{O}(5)$ & 1.01 & 2.12 & $3.066(5)$ & 154 & $1-x, 2-y,-z$ \\
\hline & $\mathrm{O}(1)-\mathrm{H}(1 \mathrm{O}) \cdots \mathrm{O}(2)$ & 0.98 & 1.64 & $2.612(4)$ & 168 & $2-x, 1-y,-z$ \\
\hline & $\mathrm{N}(2)-\mathrm{H}(2 \mathrm{~B}) \cdots \mathrm{O}(4)$ & 1.01 & 2.04 & $2.989(5)$ & 156 & $-x, 2-y,-z$ \\
\hline \multirow[t]{5}{*}{$\begin{array}{l}\text { FSM-PPZ } \\
(2: 1)\end{array}$} & $\mathrm{N}(2)-\mathrm{H}(2 \mathrm{~B}) \cdots \mathrm{O}(1)$ & 1.01 & 1.96 & $2.852(4)$ & 146 & $\begin{array}{l}-1 / 2+x, 1 / 2-y,- \\
1 / 2+z\end{array}$ \\
\hline & $\mathrm{N}(3)-\mathrm{H}(3 \mathrm{~A}) \cdots \mathrm{O}(1)$ & 1.01 & 1.72 & $2.715(3)$ & 167 & $\begin{array}{l}-1 / 2+x, 1 / 2-y,- \\
1 / 2+z\end{array}$ \\
\hline & $\mathrm{N}(3)-\mathrm{H}(3 \mathrm{~B}) \cdots \mathrm{O}(2)$ & 1.01 & 1.73 & $2.688(3)$ & 157 & $\begin{array}{l}5 / 2-x,-1 / 2+y, 3 / 2- \\
z\end{array}$ \\
\hline & $\mathrm{C}(12)-\mathrm{H}(12) \cdots \mathrm{O}(4)$ & 1.08 & 2.40 & $3.282(5)$ & 137 & $\begin{array}{l}5 / 2-x, 1 / 2+y, 3 / 2- \\
z\end{array}$ \\
\hline & $\begin{array}{l}\mathrm{C}(15)- \\
\mathrm{H}(15 \mathrm{~A}) \cdots \mathrm{O}(5)\end{array}$ & 1.08 & 2.24 & $3.155(4)$ & 141 & $2-x,-y, 1-z$ \\
\hline
\end{tabular}


Table S2. Melting points of salt/cocrystal/coformer/API.

\begin{tabular}{|l|l|l|l|}
\hline Cocrystal/salts & $\begin{array}{l}\text { Coformer MP } \\
\left({ }^{\circ} \mathrm{C}\right)\end{array}$ & API/Coformer water solubility & $\begin{array}{l}\text { Cocrystal MP } \\
\left({ }^{\circ} \mathrm{C}\right)\end{array}$ \\
\hline FSM & 206 & $<0.1 \mathrm{mg} / \mathrm{ml}$ & 206 \\
\hline $\begin{array}{l}\text { FSM-PCM } \\
\text { sesquihydrate }\end{array}$ & 105 & Soluble & $\sim 120$ \\
\hline FSM-TOL-EtOH & $161-163$ & $\sim 0.5 \mathrm{mg} / \mathrm{mL}$ & $\sim 175$ \\
\hline FSM-ANT & $109-111$ & Sparingly soluble & 144 \\
\hline FSM-TMPZ & $77-80$ & $4 \mathrm{mg} / \mathrm{mL}$ & 170 \\
\hline FSM-PPZ $(1: 1)$ & $109-112$ & $1000 \mathrm{mg} / \mathrm{mL}$ & 225 \\
\hline FSM-THP & 273 & $7.36 \mathrm{mg} / \mathrm{mL}$ & 201 \\
\hline FSM-CAF & 238 & $21.6 \mathrm{mg} / \mathrm{mL}$ & 225 \\
\hline FSM-ADN & 360 & $1.03 \mathrm{mg} / \mathrm{mL}$ & 218 \\
\hline FSM-CYT & 320 & $8 \mathrm{mg} / \mathrm{mL}$ & 232 \\
\hline FSM-PPZ $(2: 1)$ & $109-112$ & $1000 \mathrm{mg} / \mathrm{mL}$ & 235 \\
\hline
\end{tabular}

Table S3. $\mathrm{pH}$ variation after solubility experiments.

\begin{tabular}{|l|l|}
\hline Cocrystals/salts & $\mathrm{pH}($ after $1 \mathrm{~h})$ \\
\hline FSM & 6.4 \\
\hline FSM-PPZ(1:1) & 8.2 \\
\hline FSM-PPZ $(2: 1)$ & 6.9 \\
\hline FSM-TMPZ & 6.5 \\
\hline FSM-ANT & 6.3 \\
\hline FSM-CYT & 6.9 \\
\hline FSM-CAF & 6.9 \\
\hline FSM-ADE & 6.6 \\
\hline FSM-THP & 6.4 \\
\hline FSM-PCM-sesquihydrate & 6.3 \\
\hline FSM-TOL-EtOH & 6.3 \\
\hline
\end{tabular}


ORTEP diagram of FSM-TOL-MeOH

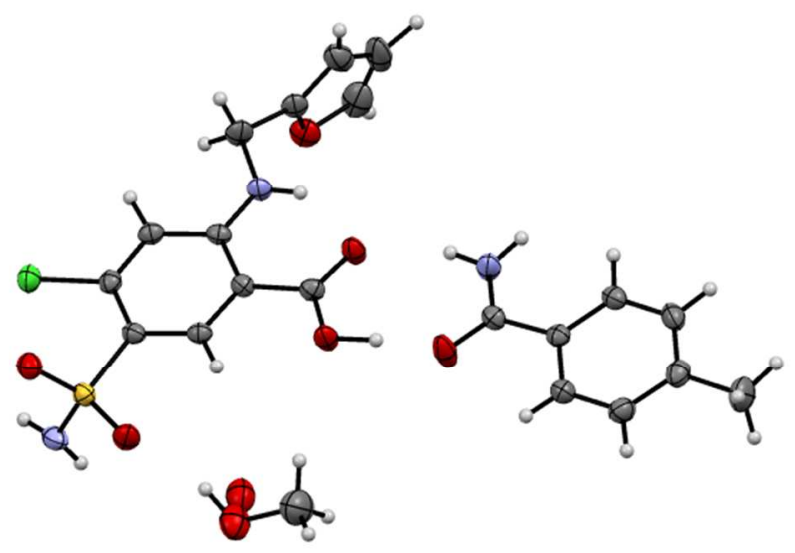

(a)

ORTEP diagram of FSM-TOL-MeNO 2

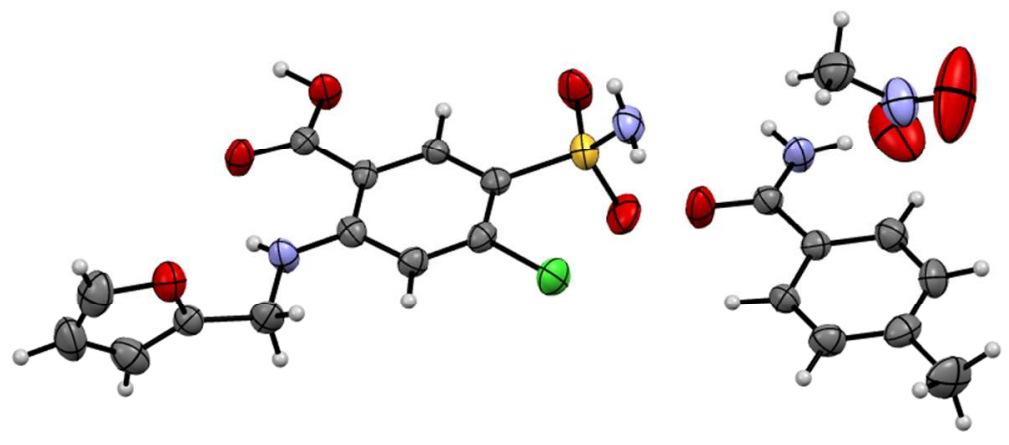

(b)

ORTEP diagram of FSM-Toluene

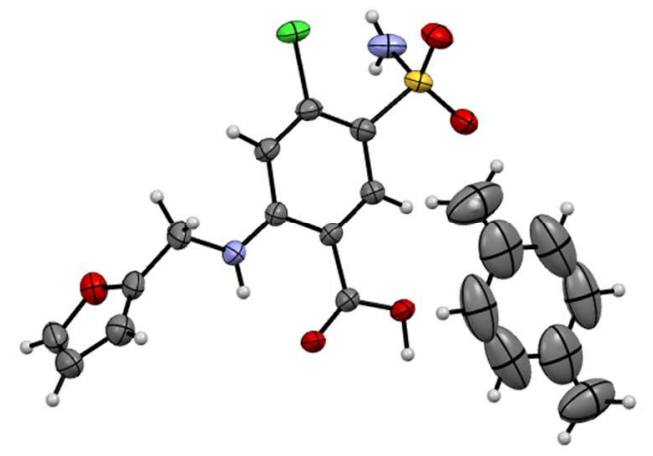

(c)

Figure S1. ORTEP diagrams of FSM-TOL-MeOH, FSM-TOL-MeNO 2 and FSM-toluene. 
FSM-TMPZ

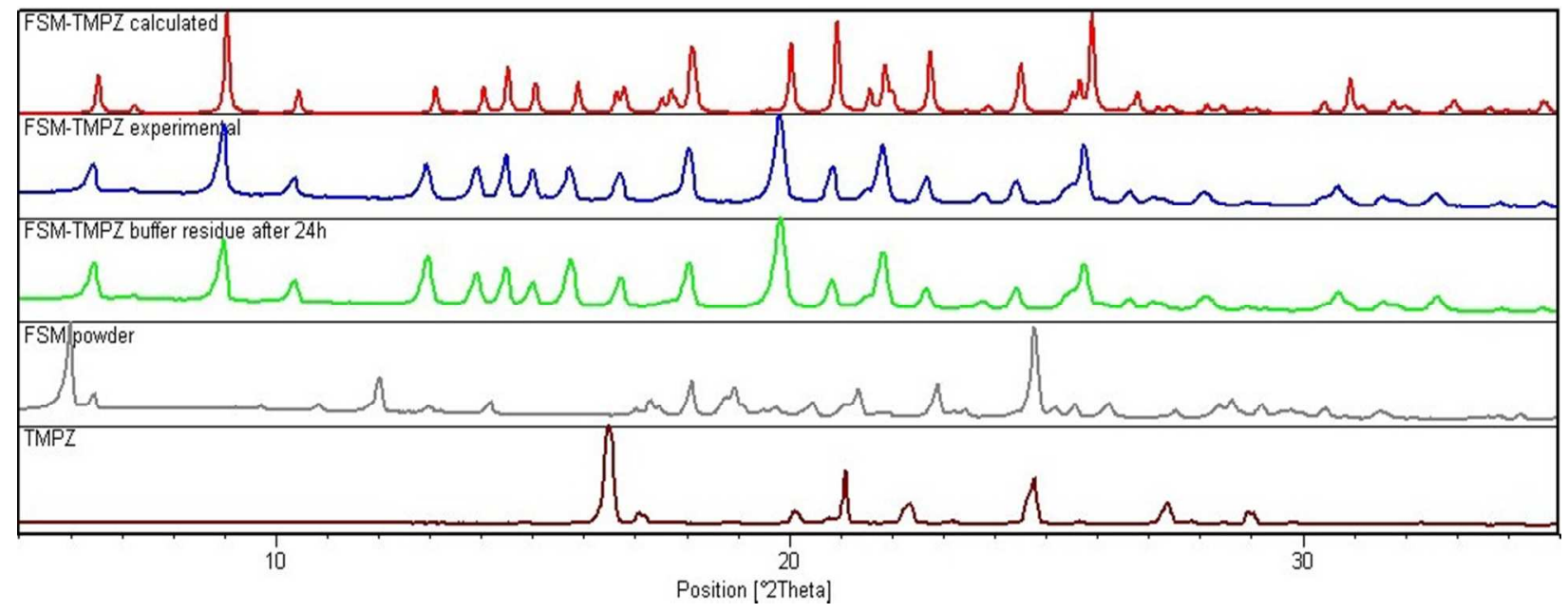

(a)

\section{FSM-ANT}

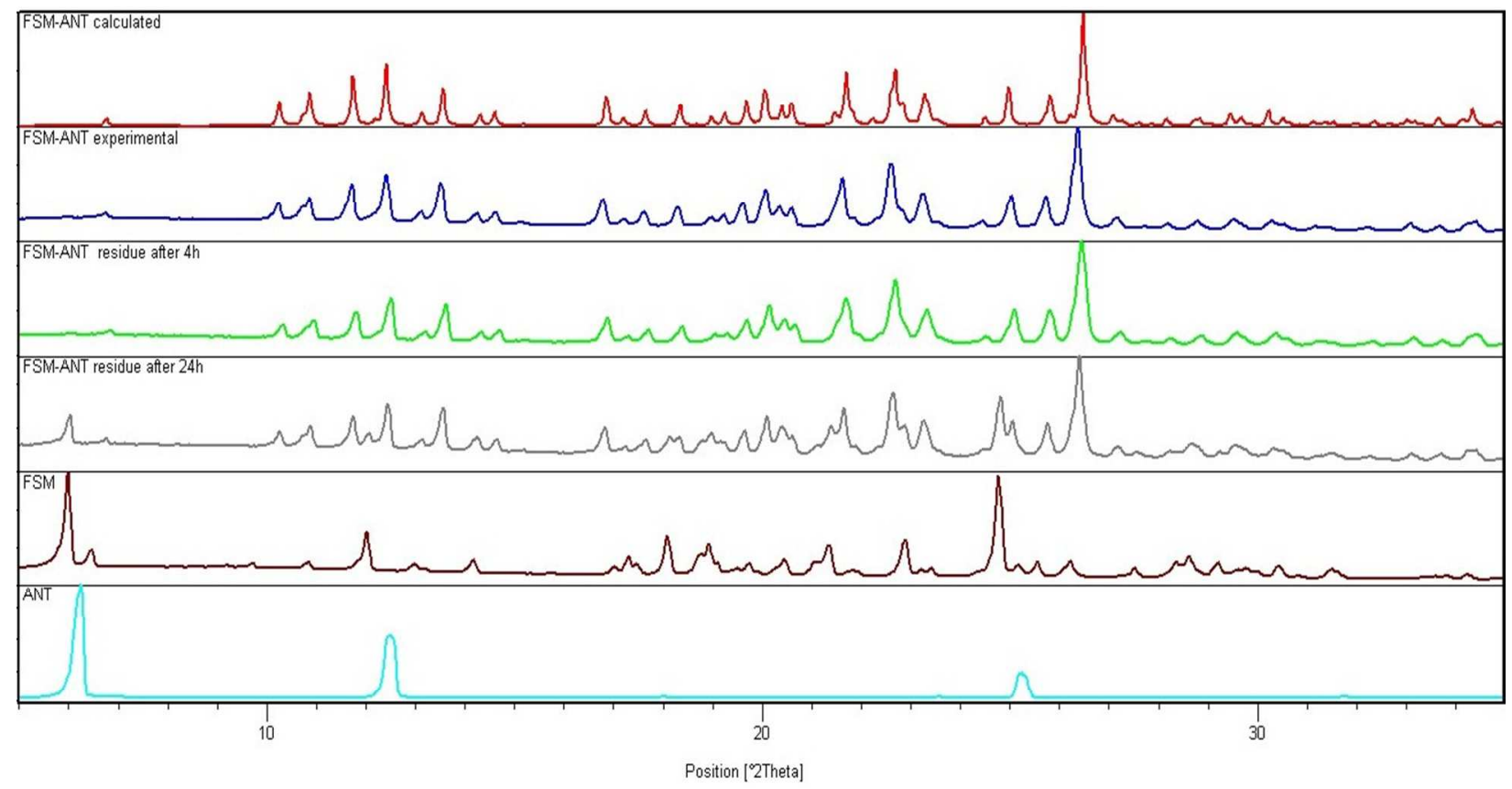

(b) 
FSM-PPZ (1:1)

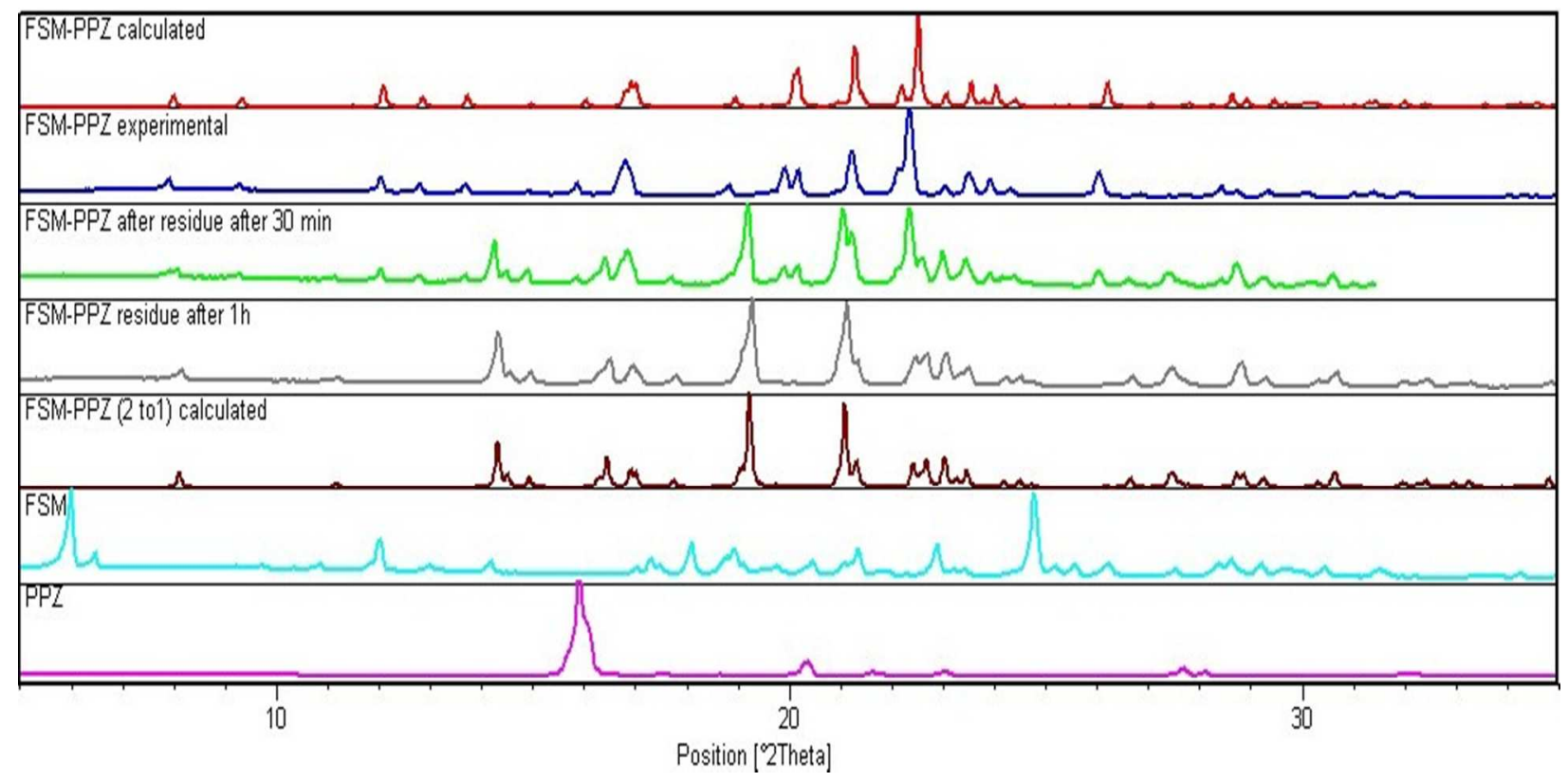

(c)

\section{FSM-TOL-EtOH}

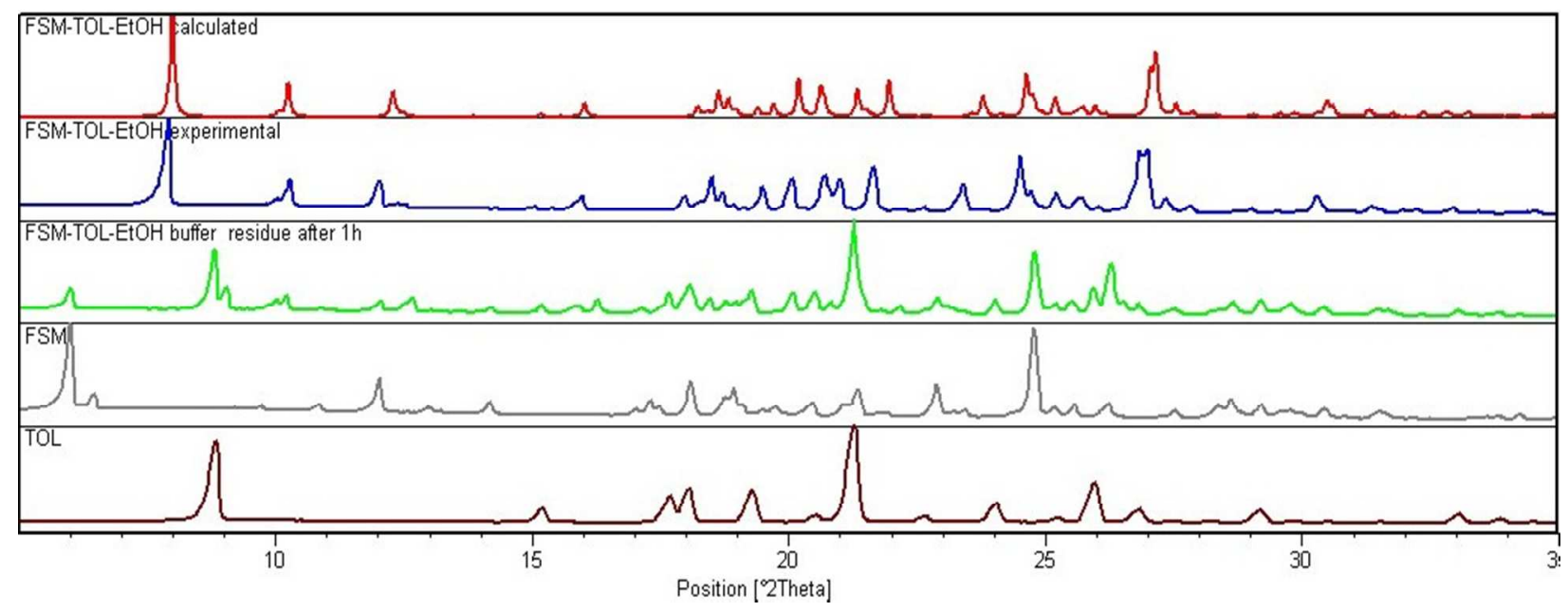

(d) 
FSM-PCM hydrate

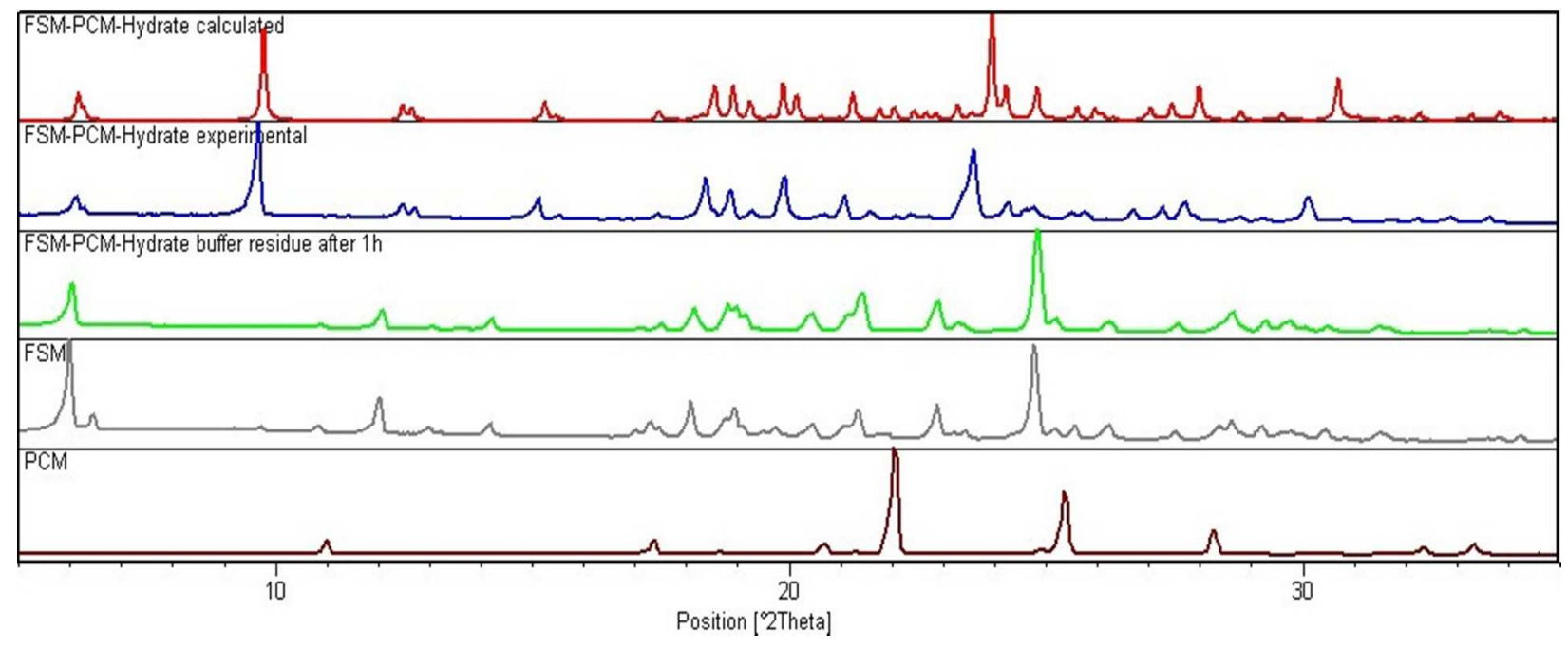

(e)

FSM-THP

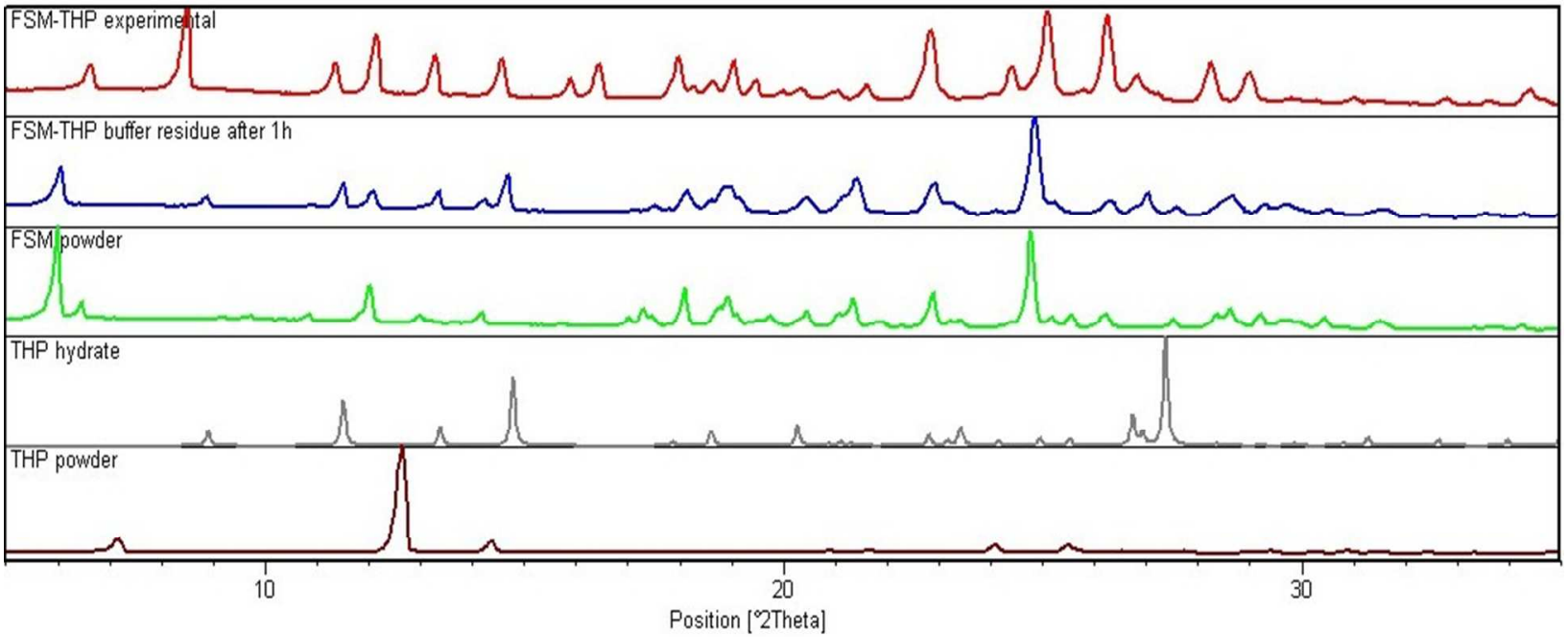

(f) 
FSM-PIC

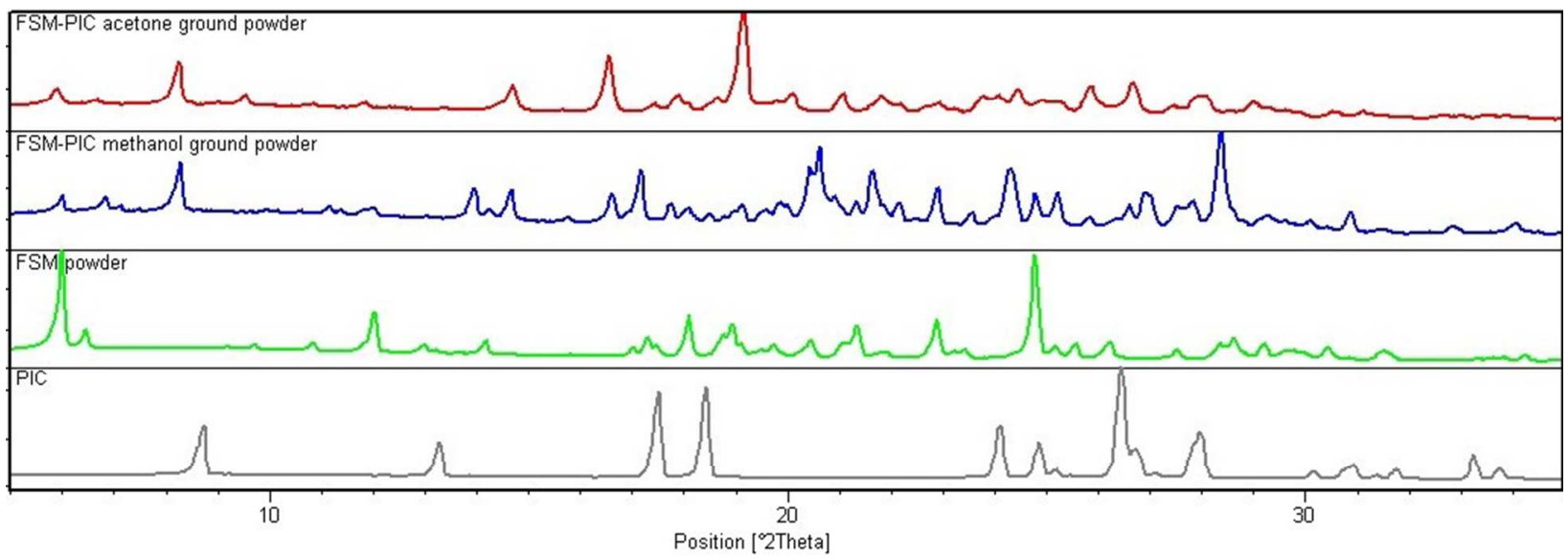

(g)

\section{FSM-INZ}

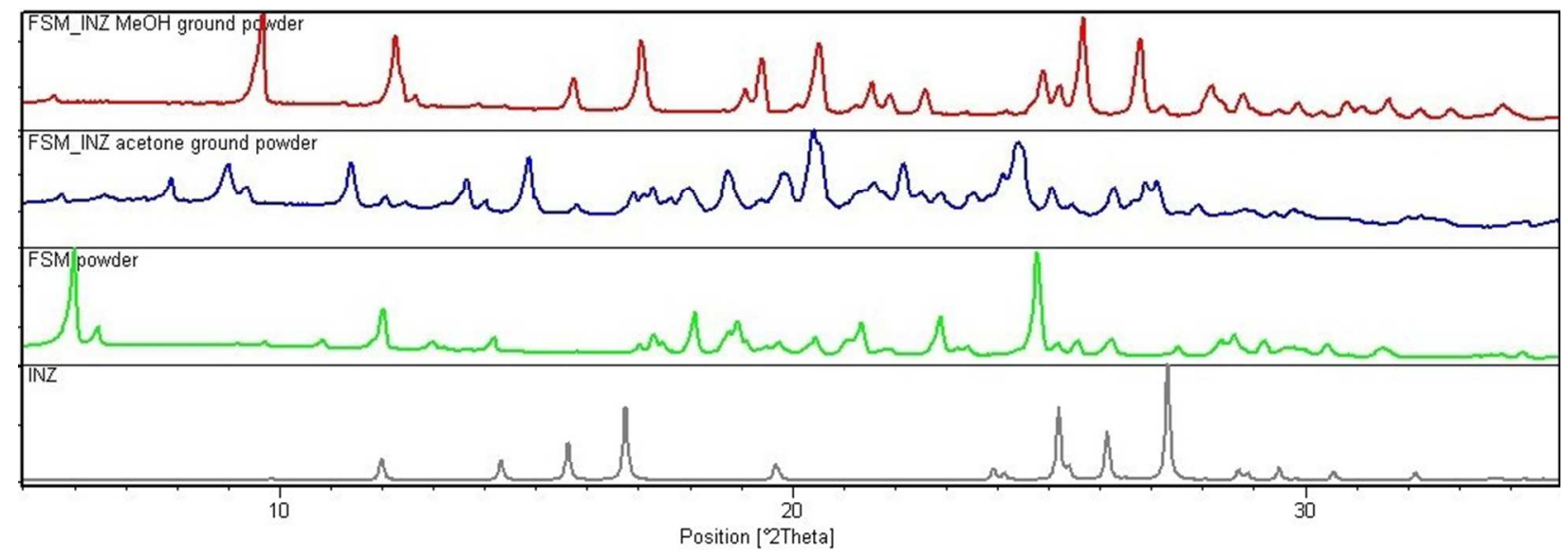

(h) 
FSM-PYZ

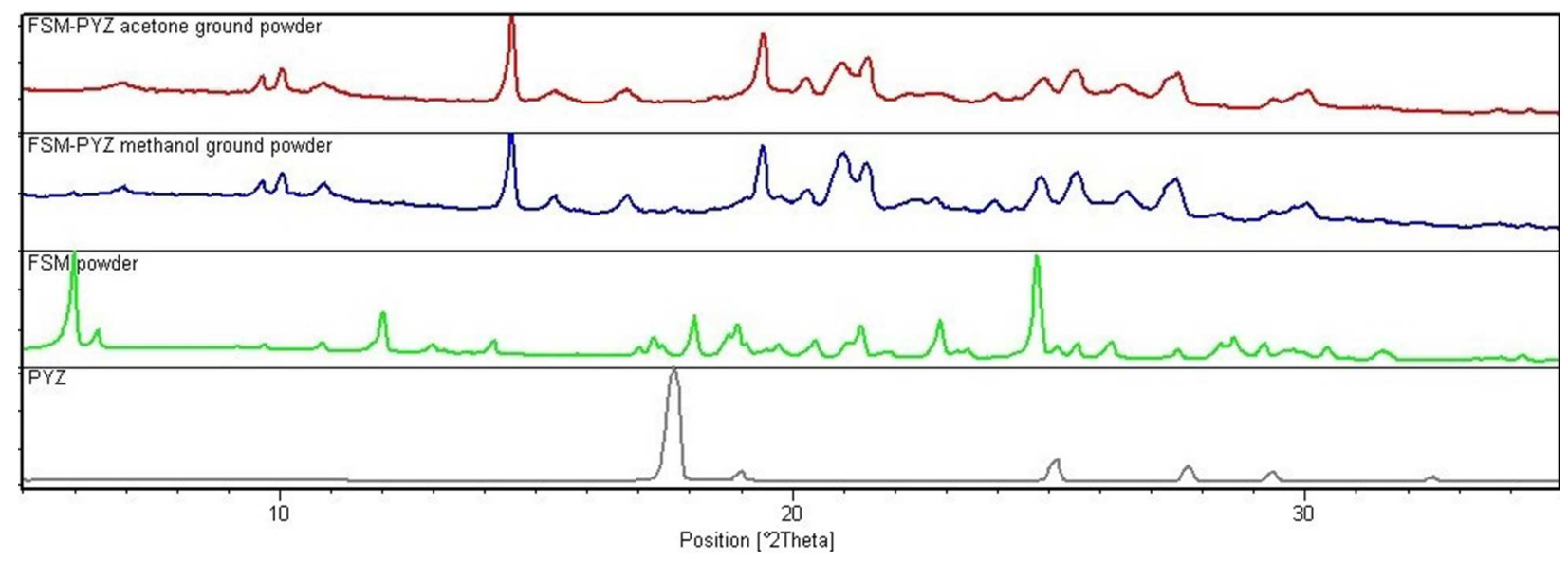

(i)

FSM-PPZ (2:1)

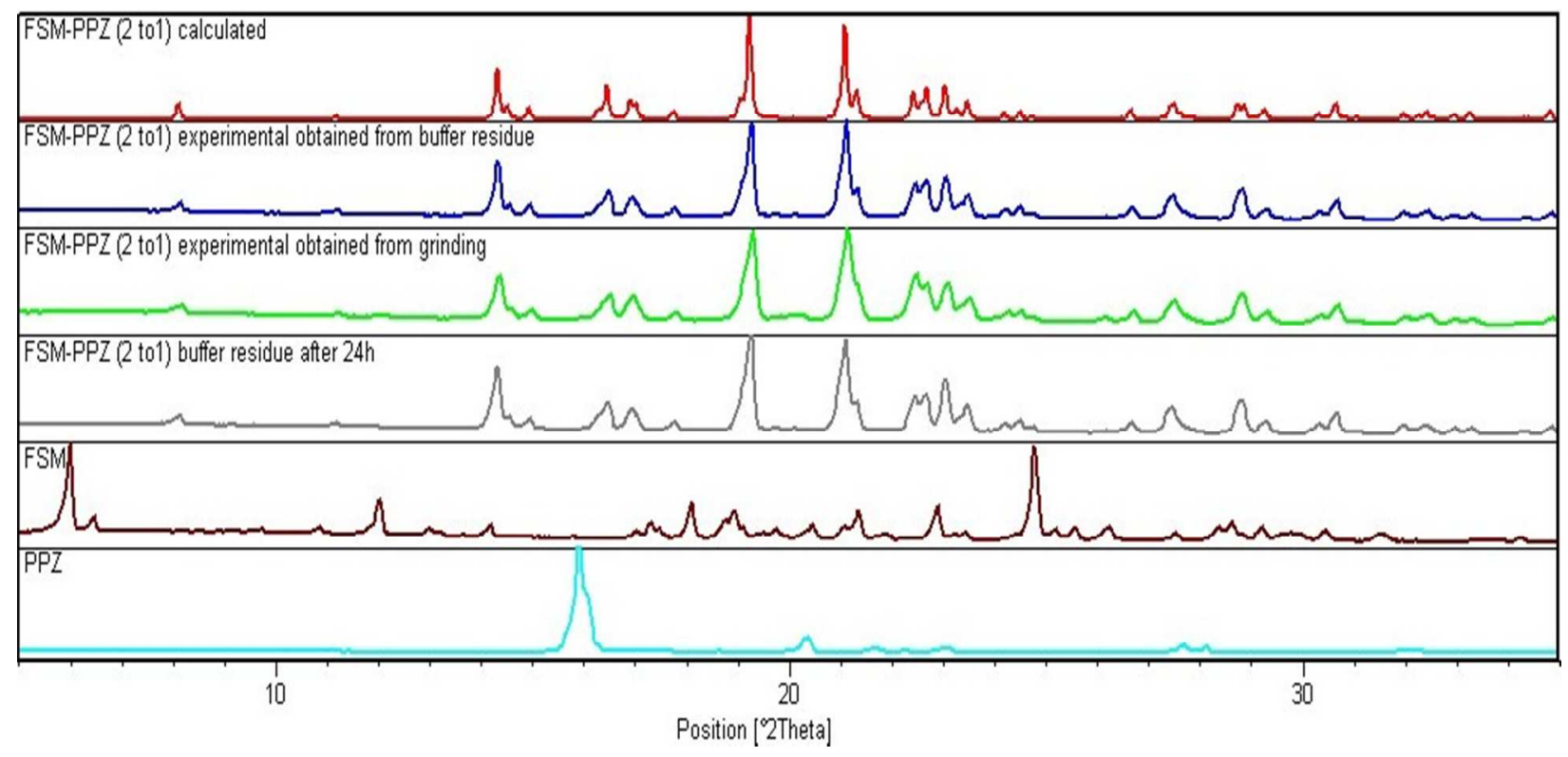

Figure S2. Powder x-ray diffraction patterns of new solid forms of FSM. 


\section{FSM-TMPZ}

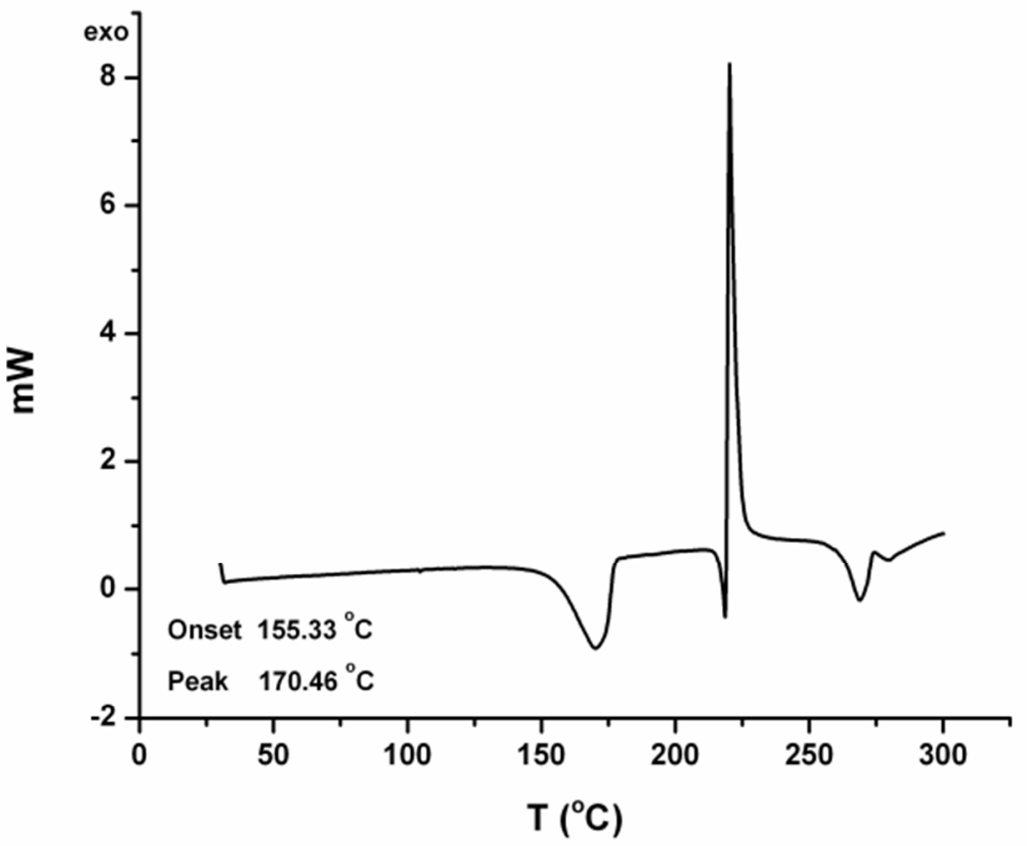

(a)

\section{FSM-ANT}

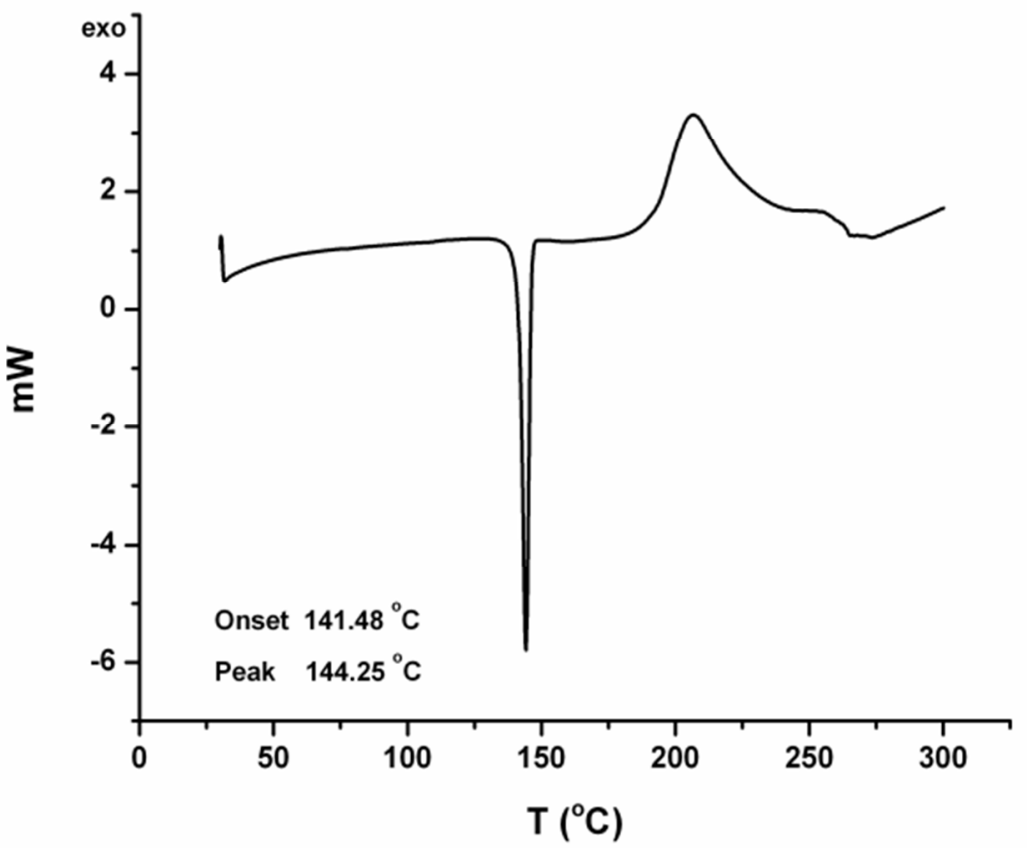

(b) 


\section{FSM-PPZ}

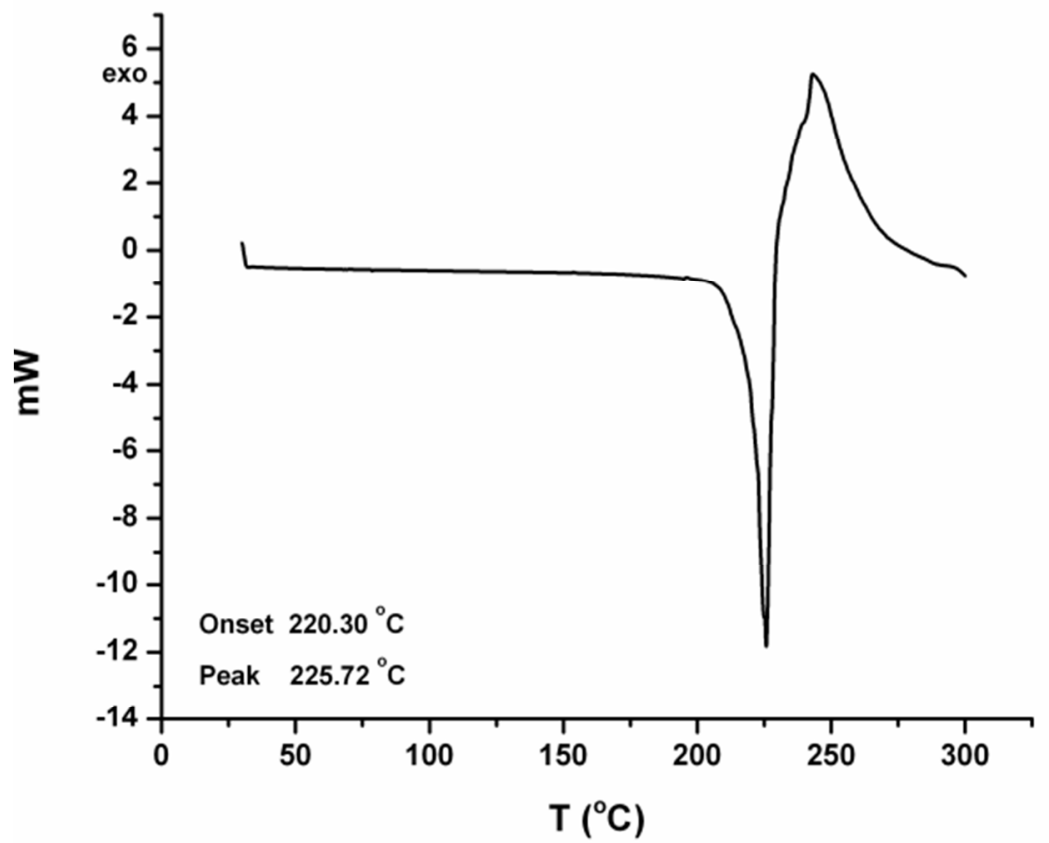

(c)

\section{FSM-TOL-EtOH}

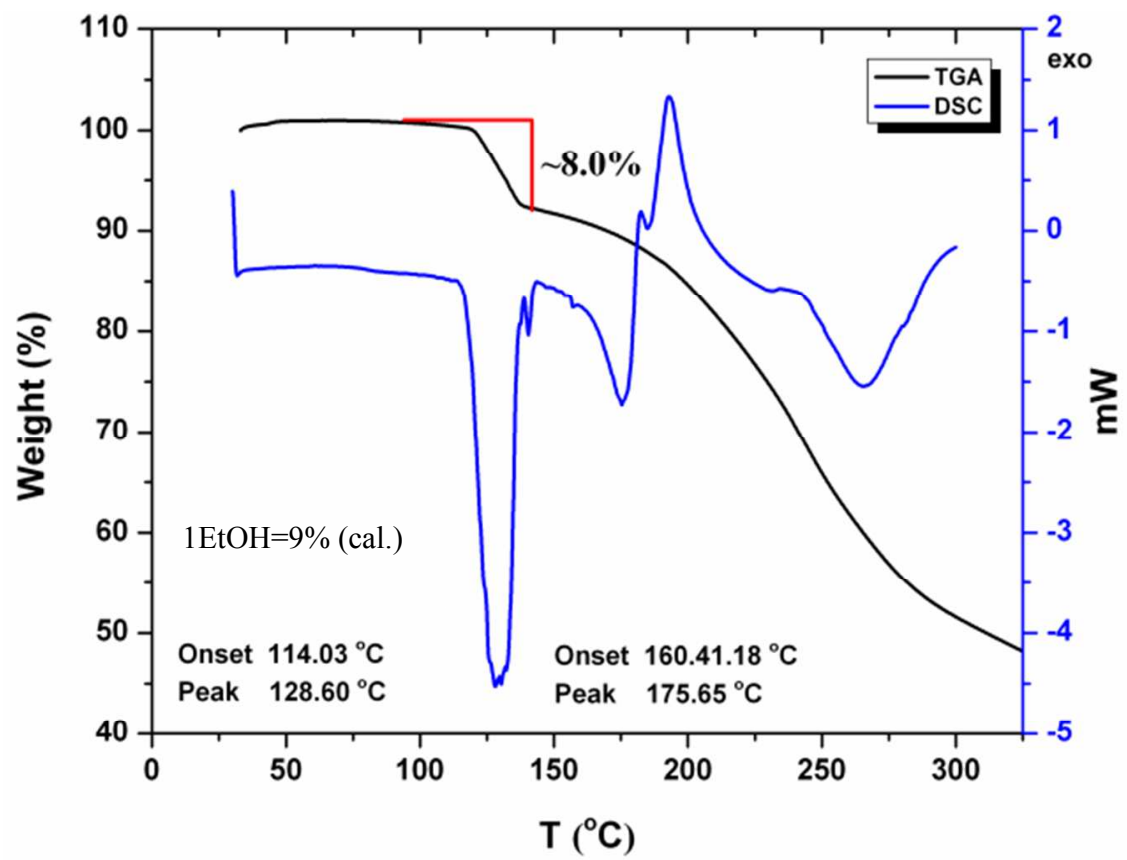

(d) 
FSM-PCM sesquihydrate

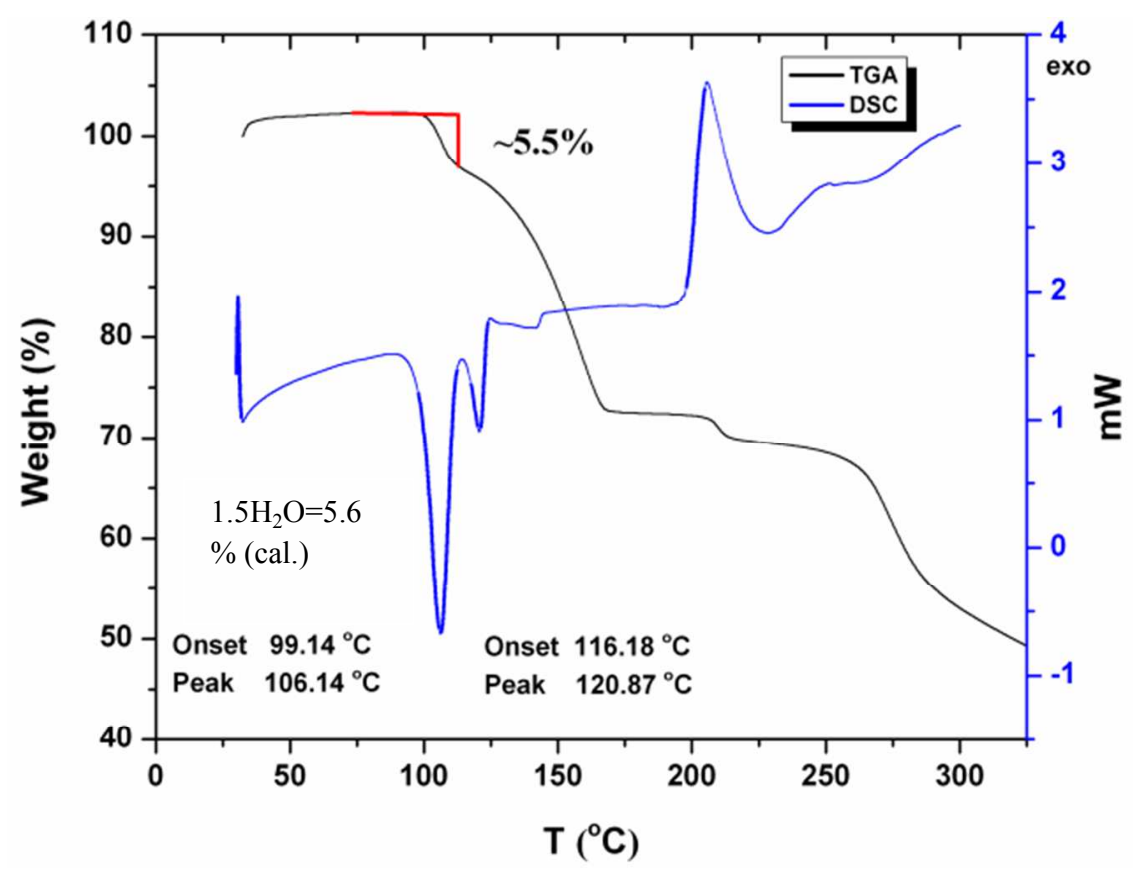

(e)

\section{FSM-THP}

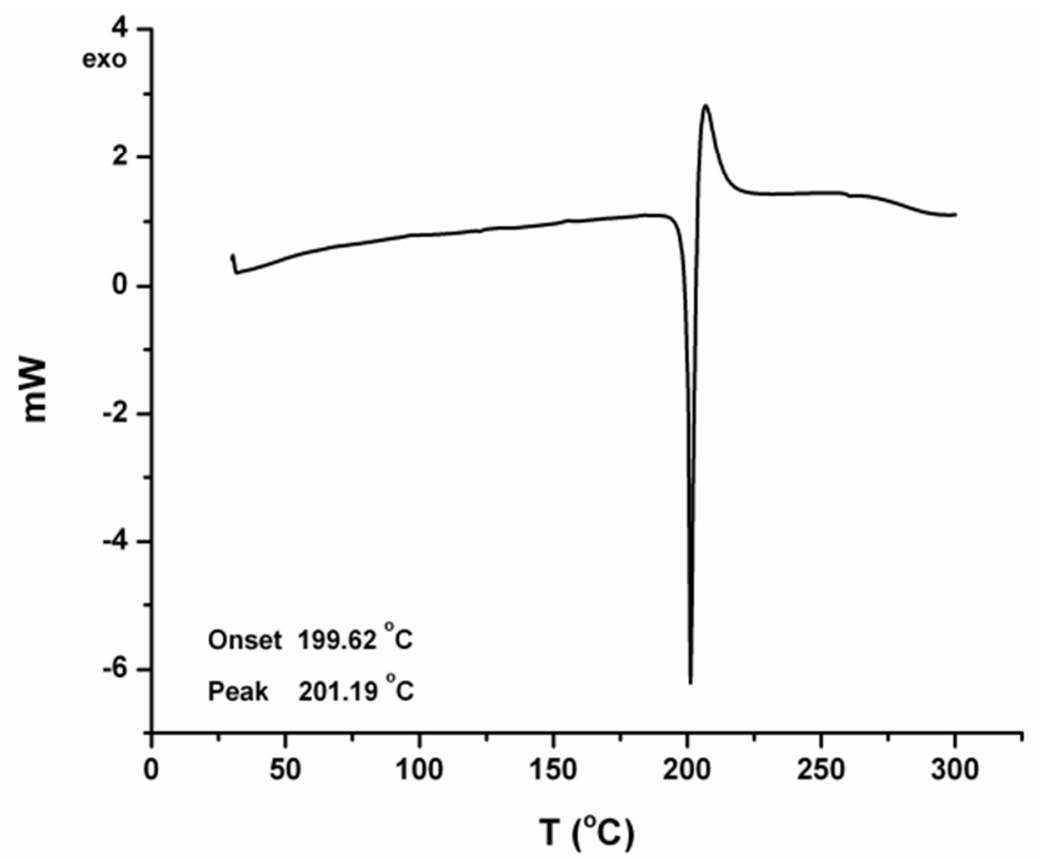

(f) 


\section{FSM}

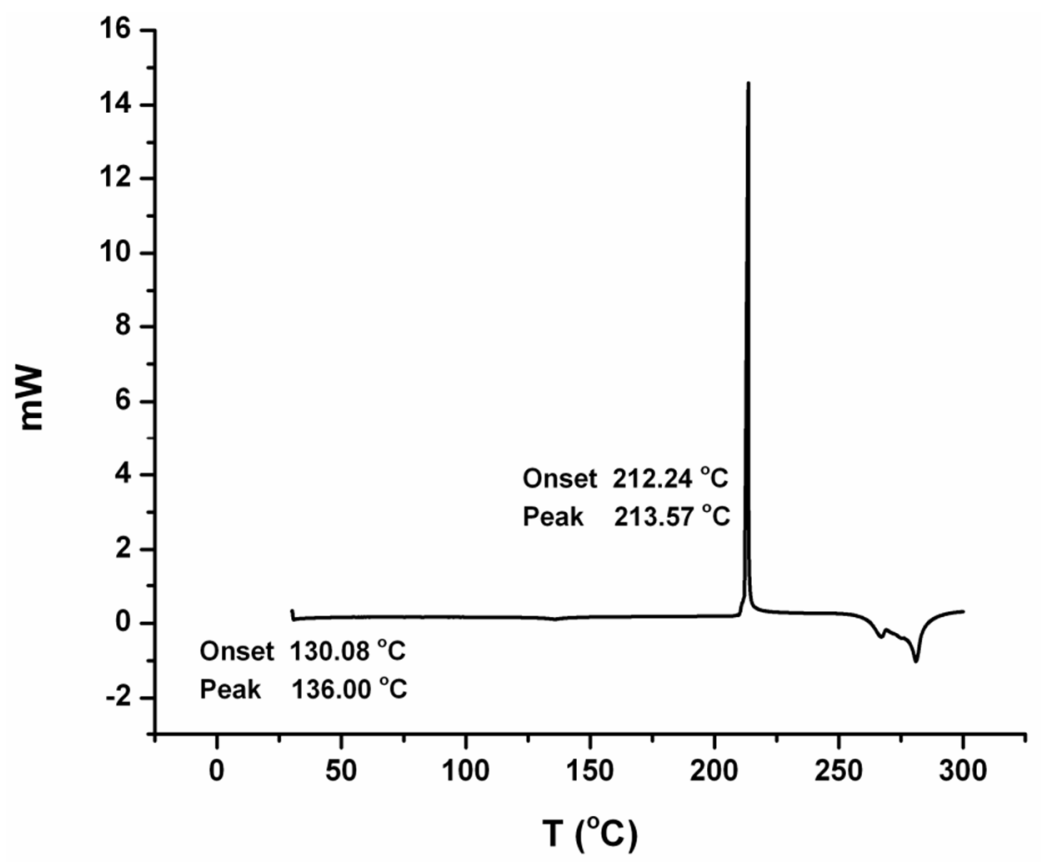

$(\mathrm{g})$

Figure S3. DSC and TGA of FSM and its salt/cocrystals.

\section{FSM-TMPZ}

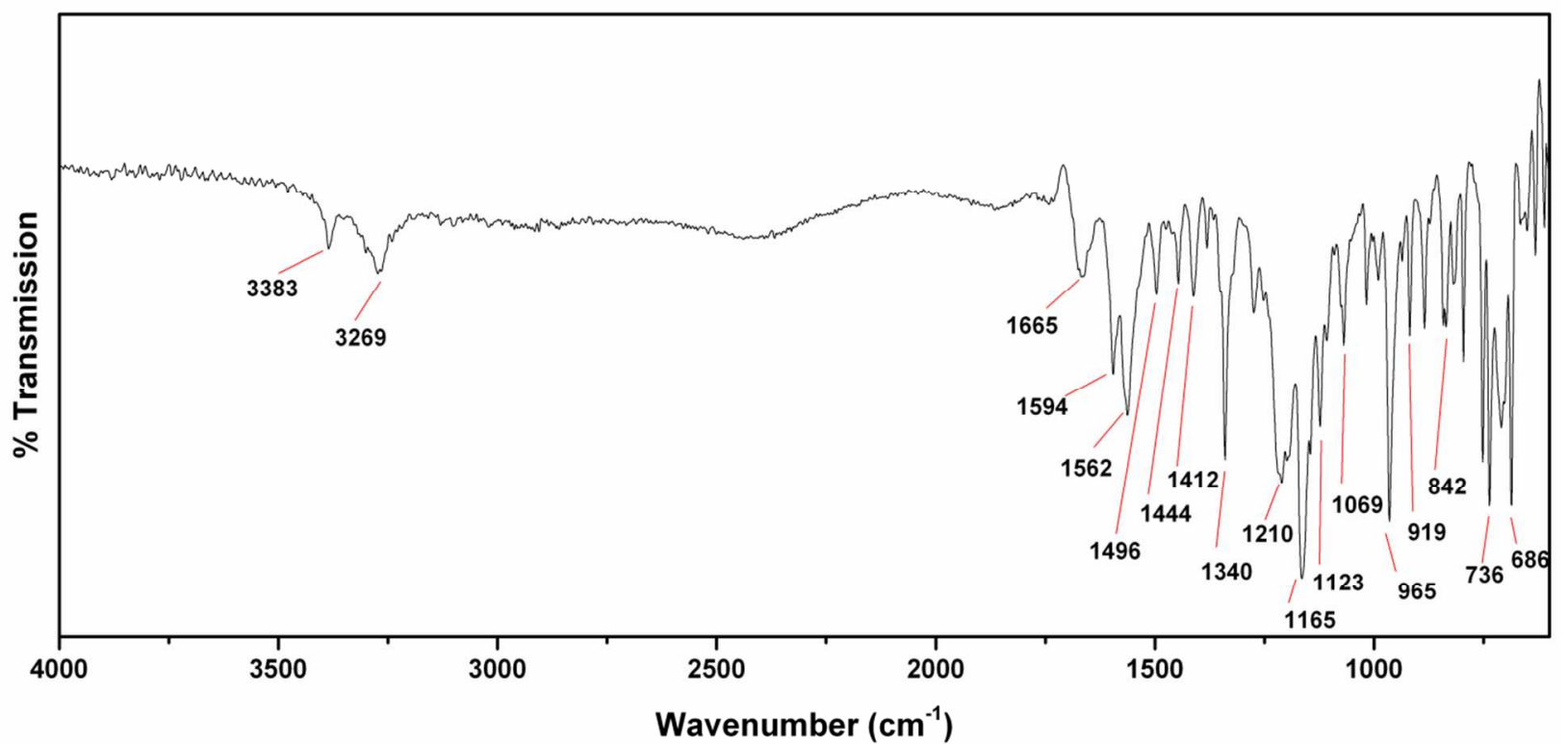

(a) 


\section{FSM-ANT}

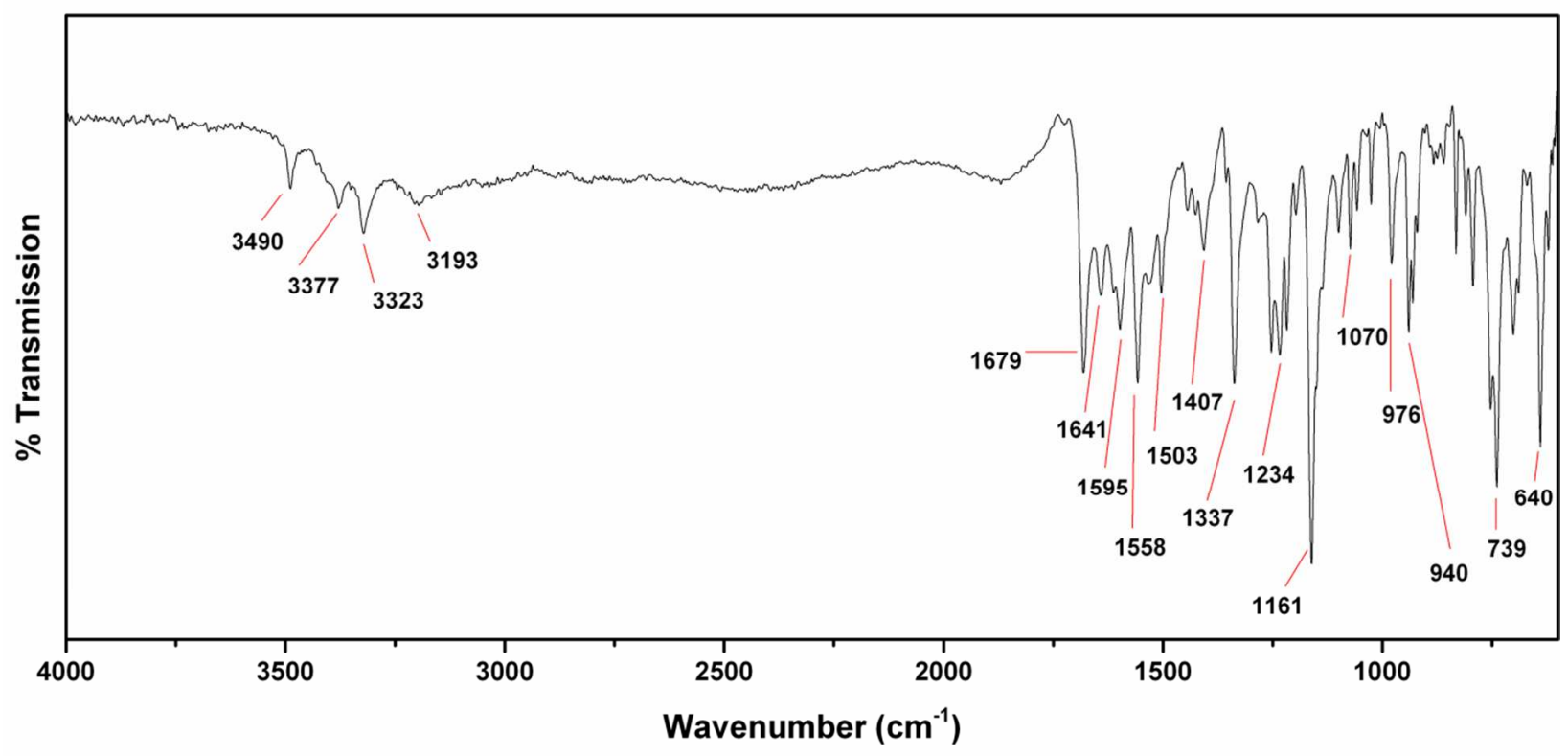

(b)

\section{FSM-PPZ}

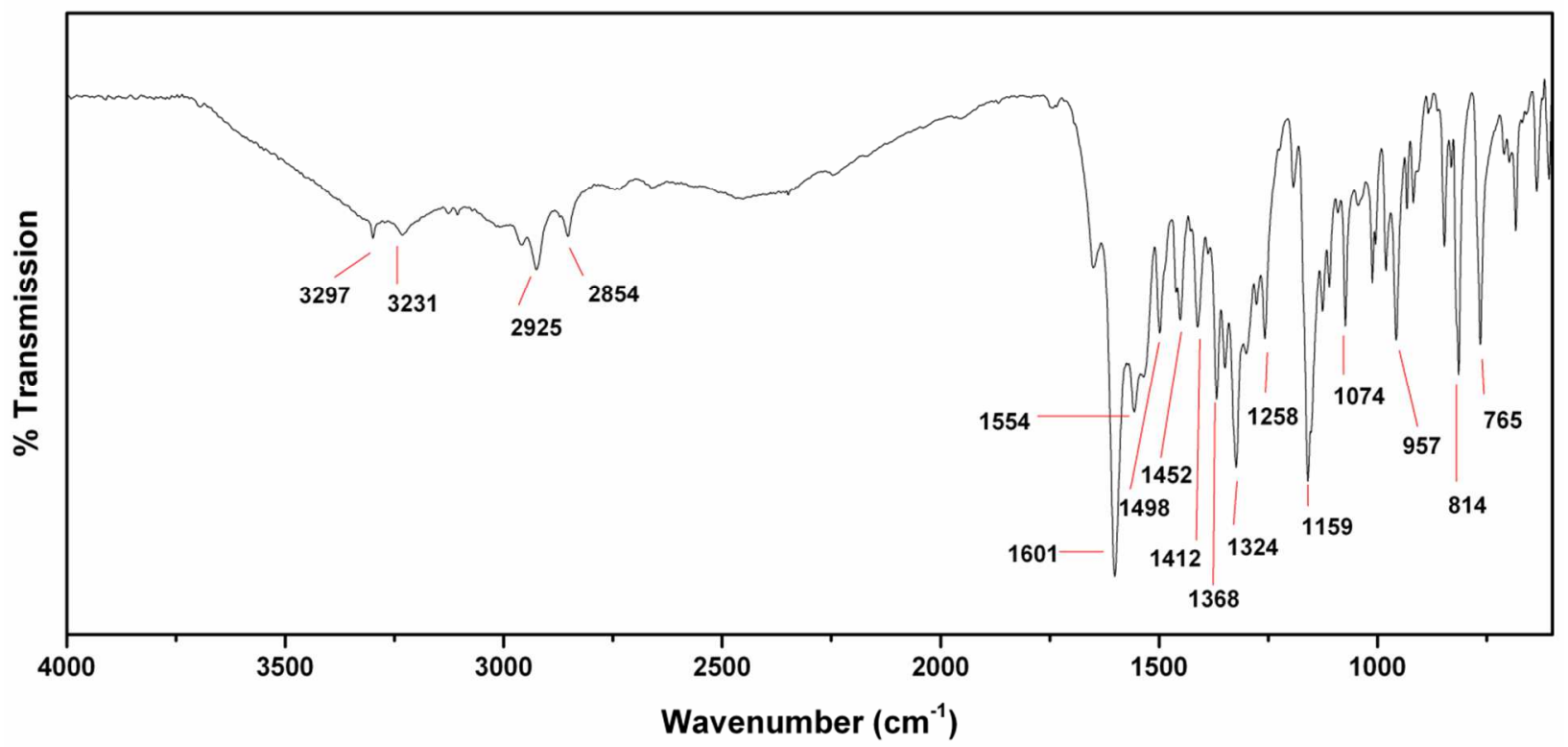

(c) 
FSM-TOL-EtOH

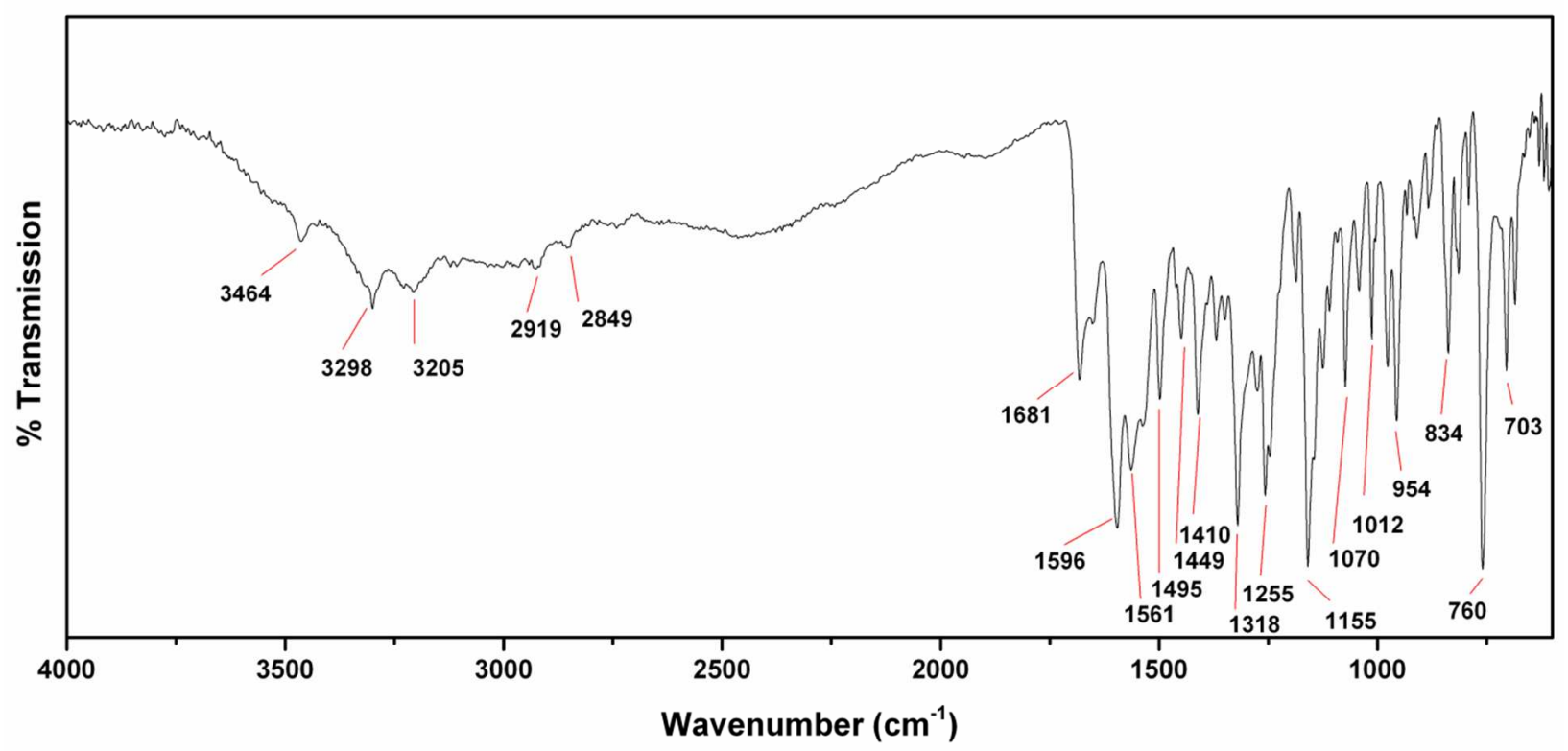

(d)

FSM-PCM sesquihydrate

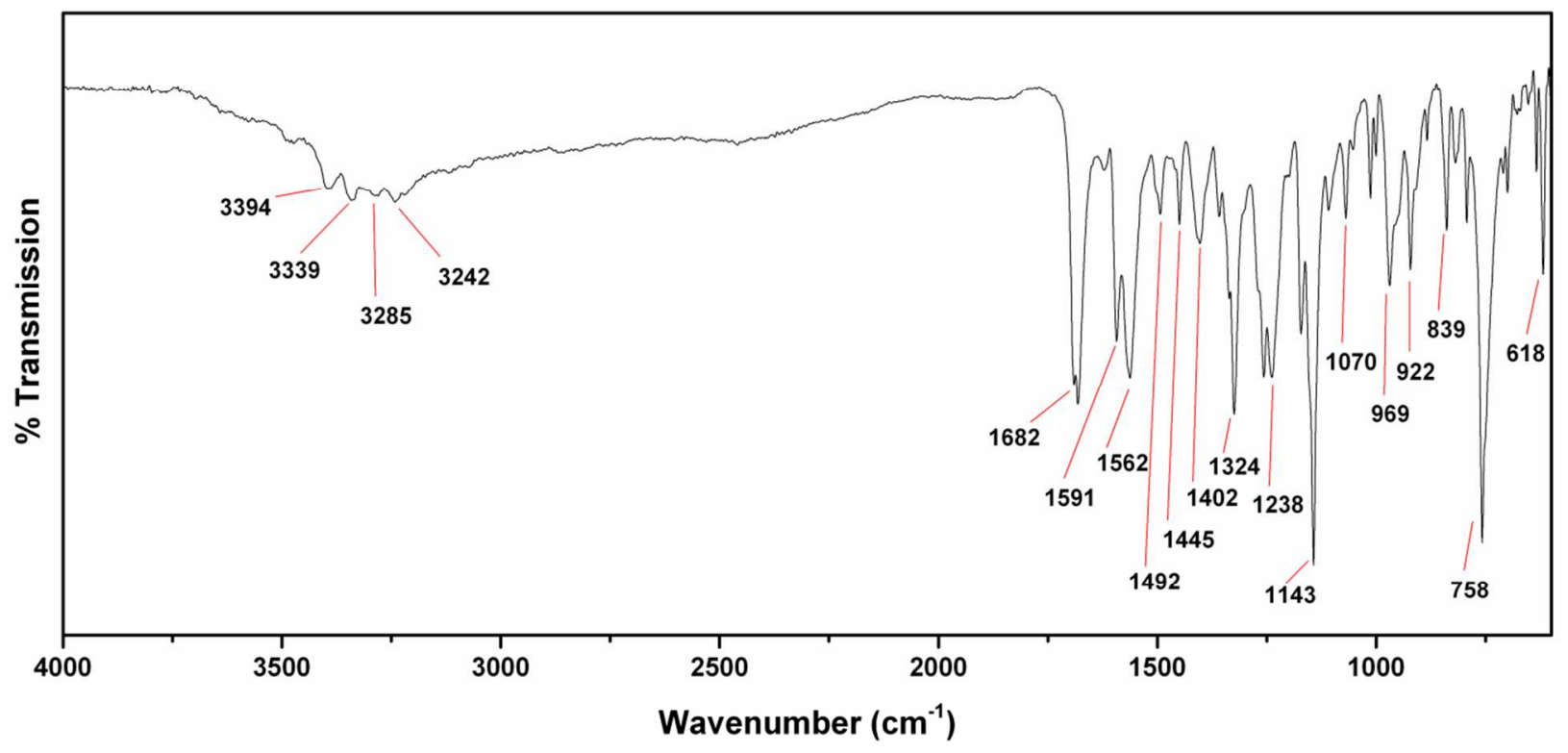

(e) 


\section{FSM-THP}

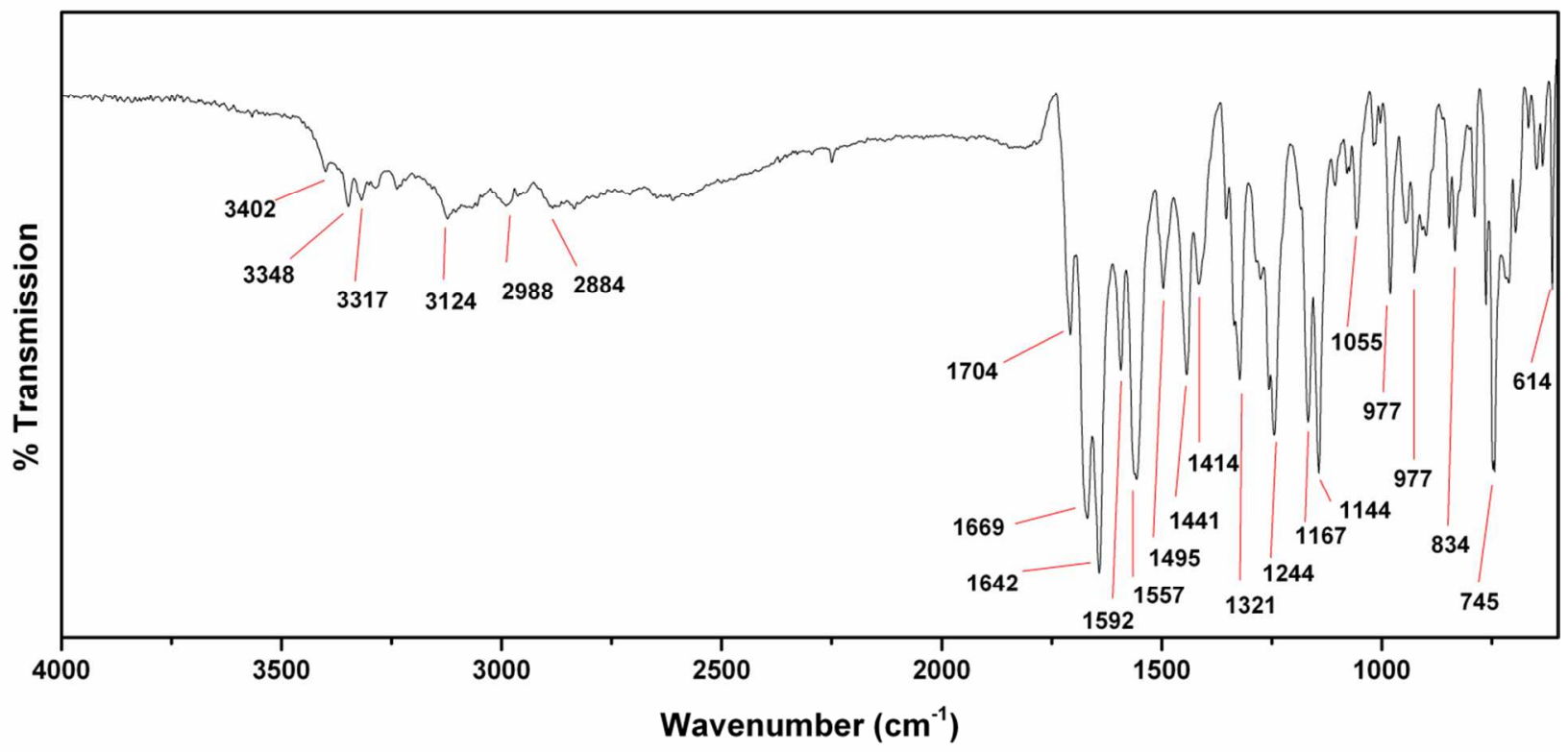

(f)

\section{FSM}

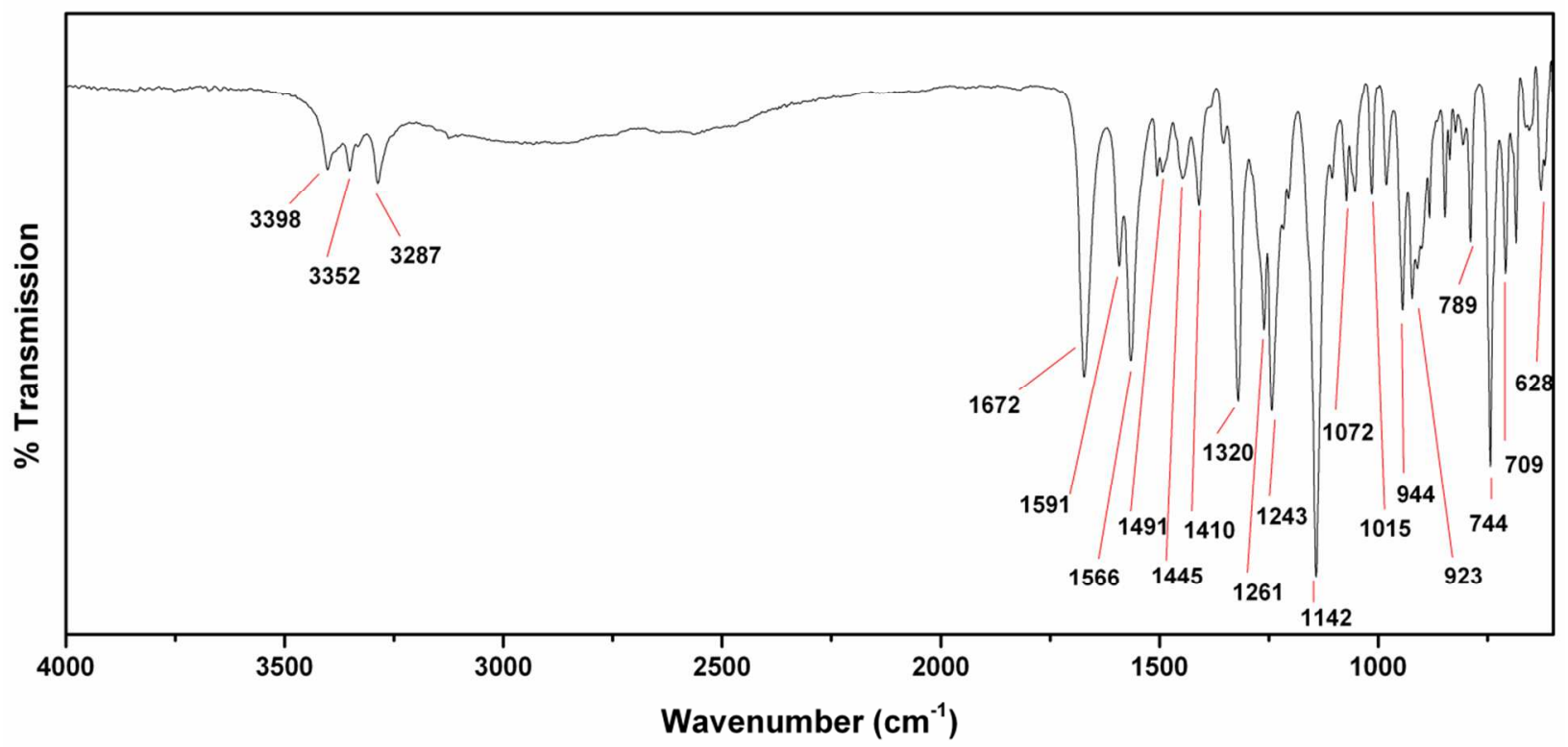

(g)

Figure S4. FT-IR spectra of FSM and its salt/cocrystals. 


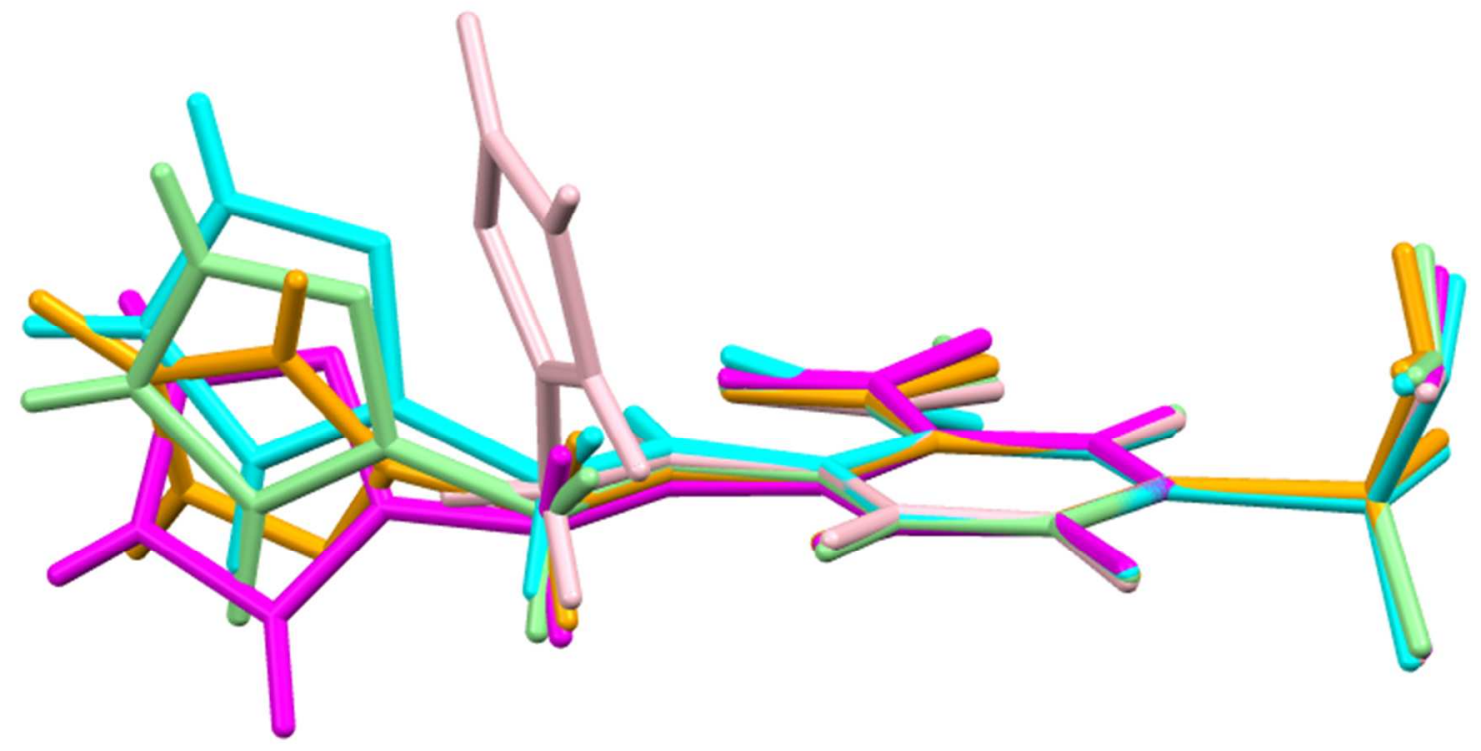

Figure S5. Overlay diagrams of FSM conformations. (Pink: FSM-ANT, orange: FSM-TMPZ, light green: FSM-TOL-EtOH, cyan: FSM-PPZ (1:1) and magenta: FSM-PPZ (2:1); FSM-PCMsesquihydrate is not included due to disorder). 